\title{
Absorption of plant lignans from cereals in an experimental pig model
}

\author{
Anne Katrine Bolvig ${ }^{1 *}$, Herman $_{\text {Adlercreutz }}{ }^{2}$, Peter Kappel Theil ${ }^{1}$, Henry Jørgensen ${ }^{1}$ \\ and Knud Erik Bach Knudsen ${ }^{1}$ \\ ${ }^{1}$ Department of Animal Science, Aarbus University, Blichers Allé 20, DK-8830 Tjele, Denmark \\ ${ }^{2}$ Nutrition and Cancer Folkhälsan Research Center and Division of Clinical Chemistry, Institute for Preventive Medicine, \\ Biomedicum Helsinki, University of Helsinki, PO Box 63, FIN-O0014 Helsinki, Finland
}

(Submitted 4 August 2015 - Final revision received 8 February 2016 - Accepted 9 February 2016 - First published online 22 March 2016)

\section{Abstract}

Plant lignans are diphenolic compounds ingested with whole grains and seeds and converted to enterolignans by the colonic microbiota. In the present study, we investigated absorption and metabolism of plant lignans and enterolignans in vivo after consumption of cereal-based diets. Six pigs fitted with catheters in the mesenteric artery and portal vein and with a flow probe attached to the portal vein along with twenty pigs for quantitative collection of urine were used for this study. The animals were fed bread based on wheat flour low in plant lignans and three lignan-rich breads based on whole-wheat grain, wheat aleurone flour or rye aleurone flour. Plant lignans and enterolignans in plasma were monitored daily at fast after 0-3d of lignan-rich intake, and on the 4th day of lignan-rich intake a 10-h profile was completed. Urine samples were collected after $11 \mathrm{~d}$ of lignan-rich diet consumption. The concentrations of plant lignans were low at fast, and was $1.2-2.6 \mathrm{nmol} / \mathrm{l}$ after switching from the low-lignan diet to the lignan-rich diets. However, on the profile day, the concentration and quantitative absorption of plant lignans increased significantly from $33 \mathrm{nmol} / \mathrm{h}$ at fast to $310 \mathrm{nmol} / \mathrm{h} 0-2.5 \mathrm{~h}$ after ingestion with a gradual increase in the following periods. Quantitatively, the absorption of plant lignans across diets amounted to $7 \%$ of ingested plant lignans, whereas the urinary excretion of plant lignans was $3 \%$ across diets. In conclusion, there is a substantial postprandial uptake of plant lignans from cereals, suggesting that plant lignans are absorbed from the small intestine.

Key words: Lignans: Cereals: Absorption of lignans: Plasma: Catheterised pigs

Epidemiological studies have linked the intake of plant lignans with protection against a number of lifestyle-related diseases such as certain cancers, CVD, type 2 diabetes and hormonally induced diseases $^{(1,2)}$. Intervention ${ }^{(3-6)}$, cross-sectional $^{(7-9)}$ and animal studies $^{(10,11)}$ have shown a positive relationship between the intake of plant lignans and plasma levels of enterolignans, and the health benefits linking high lignan intake and the accompanying high enterolignan serum concentration have been known for more than a decade ${ }^{(1,12)}$. The biological activity of enterolignans may be related to their oestrogen-like structure, and enterolignans have been found to bind weakly to oestrogen receptors ${ }^{(13)}$ and sex-hormone binding globulins (SHBG) ${ }^{(14)}$ and to be positively associated with levels of $\mathrm{SHBG}^{(15)}$. Moreover, lignans have been shown to inhibit 5- $\alpha$-reductase ${ }^{(16)}$, reduce insulin-growth factor $1^{(17)}$ and to suppress fatty acid synthase protein expression ${ }^{(18)}$.

In the Nordic countries, whole-grain cereals, fruits and vegetables are the main sources of plant lignans. Plant lignans consist of a number of non-nutritive diphenolic compounds identified as follows: secoisolaricinol (SECO), matairesinol
(MAT), lariciresinol (LAR), pinoresinol (PIN), medioresinol and syringaresinol $^{(19,20)}$. The conventional view is that plant lignans are converted to enterolignans (enterolactone (ENL) and enterodiol (END)) by the colonic microbiota and are absorbed through the colonic barrier ${ }^{(1)}$, which has the capability to reconjugate enterolignans. Studies performed with the lignan secoisolariciresinol diglucoside (SDG), the most abundant lignan in flaxseed, have further shown that, although END are produced by dominant members of the gut microbial community, it is the subdominant species that are responsible for the production of $\mathrm{ENL}^{(21,22)}$.

Numerous studies have dealt with the metabolism of plant lignans to enterolignans; however, only a few have investigated the absorption and functional role of plant lignans. Kinetic studies show that plant lignans provided as SDG and sesamin are rapidly absorbed presumably from the small intestine and appear in the systemic circulation within hours after ingestion ${ }^{(19,23)}$; 3 h after consumption of cereals, plant lignans have been found in plasma from pigs ${ }^{(11)}$; however, the kinetics

Abbreviations: LAR, lariciresinol; MAT, matairesinol; PIN, pinoresinol; RAF, rye aleurone-rich flour; SDG, secoisolariciresinol diglucoside; SECO, secoisolaricinol; WAF, wheat aleurone-rich flour; WFL, wheat flour; WWG, wheat-whole grain.

* Corresponding author: A. K. Bolvig, fax +458715 4247, email annek.sorensen@anis.au.dk

* Deceased. 
Table 1. Ingredient list of the diets

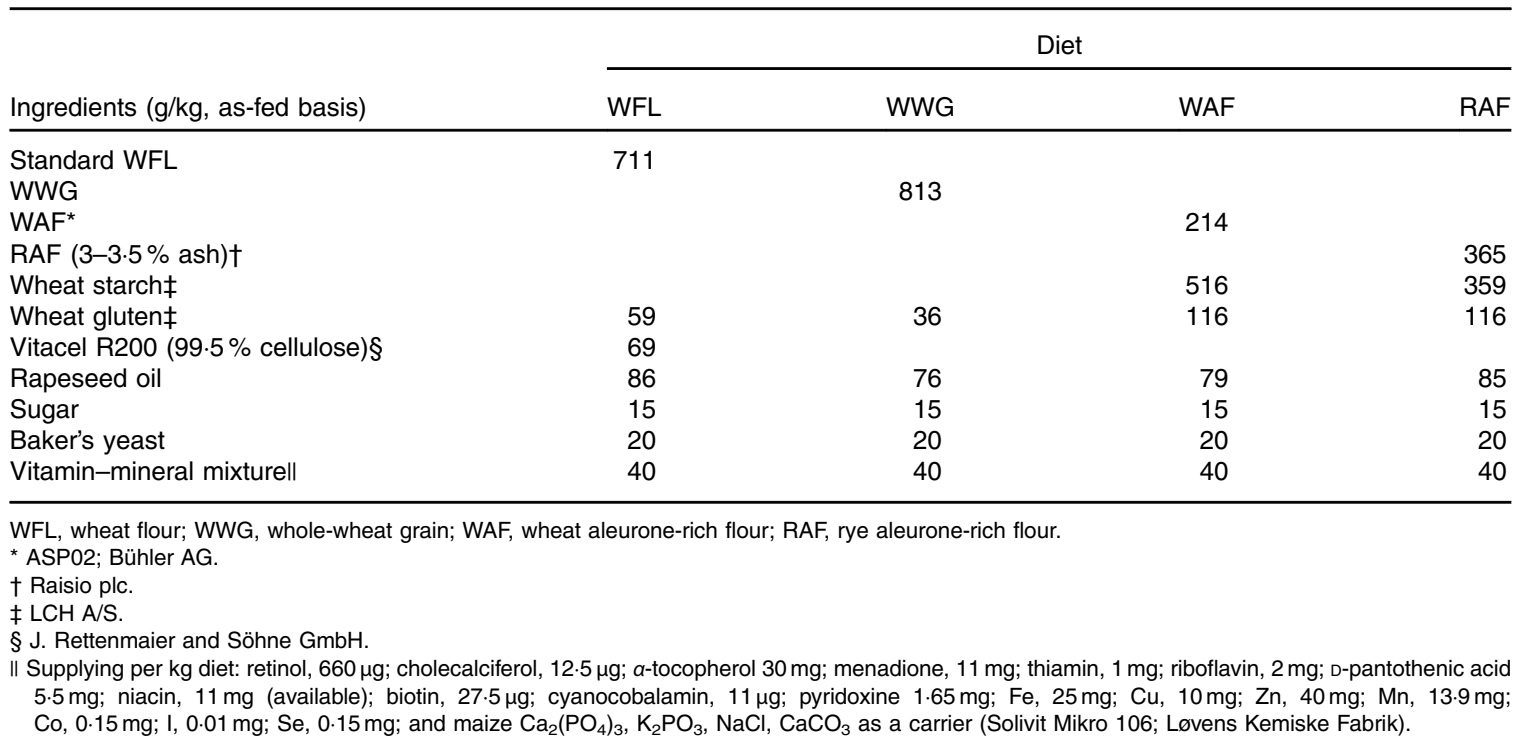

or mechanism of uptake of plant lignans from cereals has not been studied. In cereals, plant lignans are not equally distributed over the grain: the lowest concentration is found in endosperm tissue and the highest in the fibre-rich pericarp/testa and the aleurone layers ${ }^{(2)}$.

Owing to the health-beneficial effects of plant lignans, there is growing interest in understanding the dynamics behind absorption, circulation and excretion of plant lignans and enterolignans in organisms. In the present study, we investigated the effects of feeding cereal-based breads prepared from whole-grain wheat, wheat aleurone-enriched and rye aleurone-enriched fraction with focus on the diurnal variation in plant lignans and enterolignans using catheterised pigs as a model for humans. We hypothesised that plant lignans in cereals, such as the case for plant lignans sesamin and SDG, are absorbed in the small intestine.

\section{Methods}

\section{Diets}

Four bread-based diets were prepared from standard wheat flour (WFL), wheat-whole grain (WWG), wheat aleurone-rich flour (WAF) and rye aleurone-rich flour (RAF) (Table 1). Comparable dietary fibre content in the diets was obtained by addition of purified wheat fibres (Vitacel R200; J. Rettenmaier and Söhne $\mathrm{GmbH}$ ) to the WFL diet; furthermore, the diets were balanced to have similar levels of fat, protein, starch, energy, vitamins and minerals. After production, the breads were cut into pieces and meal portions were frozen at $-20^{\circ} \mathrm{C}$ and thawed immediately before consumption.

\section{Experimental design}

The pigs used in the following studies were from the swineherd at Aarhus University, Department of Animal Science, Foulum, Denmark. The Danish Animal Inspectorate approved the animal experiments, and housing and rearing were in compliance with the guidelines of the Ministry of Justice, Animal Testing Act (Consolidation Act no. 726 of 9 September 1993 as amended by Act no. 1081 of 20 December 1995), concerning animal experiments and care of animals under study. The animals were kept in individual pens $(2 \times 2 \mathrm{~m})$, and an elevated plastic grid covering half of the pen allowed the pigs to rest and stay dry. The pigs had access to water ad libitum, and were allowed $45 \mathrm{~min}$ to finish ingestion of a meal before any remaining food was removed. Straw was not supplied to the pen. Once a week, the pigs were weighed and received $400 \mathrm{mg}$ Fe supplement, which was injected intramuscularly (Uniferon ${ }^{\circledR}$; Pharmacosmo A/S).

Study 1 . The purpose of study 1 was to measure the concentration and net absorption of plant lignans and enterolignans in blood samples obtained from the portal vein and the mesenteric artery. Plasma samples were collected at fast for measuring absorption from the colon and during a 10-h profile to measure absorption from the small intestine. Six female Landrace $\times$ Yorkshire pigs $(56.5($ SEM 1.8$) \mathrm{kg})$ were included in the experiment, which was designed as a repeated $3 \times 3$ Latin square design. Pigs were adapted to the pen for $5 \mathrm{~d}$ before surgery. A flow probe (Transonic 20A probe, $20 \mathrm{~mm}$; Transonic System Inc.) was surgically fitted around the portal vein to measure portal blood flow, and permanent catheters were fitted in the portal vein and the mesenteric artery as described by Jørgensen et $a l^{(24)}$. After $5-7 \mathrm{~d}$ post-surgery recovery, the animals entered a 21-d experimental period (3 consecutive experimental weeks). The animals were fed at 09.00 hours (breakfast), 14.00 hours (lunch) and 19.00 hours (dinner) with portions corresponding to 40,40 and $20 \%$ of the total daily intake, respectively. Within the experimental weeks, the WFL diet low in lignans was fed as a washout diet on days 1-3, and then pigs were fed one of the experimental lignan-rich diets (WWG, WAF, RAF) on days 4-7. On days 4-7, fasting blood 
samples (30 min before the first daily meal) were collected from the portal vein and the mesenteric artery. In addition, on day 7 , eighteen consecutive blood samples were drawn concomitantly between 0 and $10 \mathrm{~h}$ from the portal vein and the mesenteric artery. Owing to limited blood sample volume, four artery and four venous plasma samples were pooled from 9-ml blood samples to represent the mean content of plant lignans and enterolignans in blood during the following periods: $0-2.5 \mathrm{~h}$ (Phase I), 2.5-5h (Phase II), 5-7.5 h (Phase III) and 7.5-10 h (Phase IV) after the morning feeding, respectively. Blood samples were collected in $\mathrm{Na}$ heparin vacutainers, and blood loss was replaced with saline. Blood samples were stored at $-20^{\circ} \mathrm{C}$ immediately after sample collection, and permanently stored at $-80^{\circ} \mathrm{C}$. Packed cell volume was monitored once per hour and was always $>26 \%$. Blood flow in the portal vein was recorded immediately before blood collection as a mean of sixty recordings within a minute.

Study 2. The main purpose of study 2 was to quantify the urinary excretion of plant lignans and enterolignans. A total of twenty cross-breed (Duroc $\times$ Landrace $\times$ Yorkshire) growing female pigs were ileal cannulated as described by Jørgensen et $a l .{ }^{(24)}$ (body weight at surgery: 64.9 (SEM 1.2) kg). Ileal cannulation was used in the study by Le Gall et al. ${ }^{(25)}$; however, in the present study, only urine samples were analysed. Before surgery, the pigs were fed a diet with low dietary fibre. After a $10-\mathrm{d}$ recovery period, pigs were fed the same diet as before the surgery. Four groups (five pigs in each) were formed and assigned the WFL, WWG, WAF or RAF diets. The pigs were gradually introduced to the new diets during days $7-10$ by mixing a smaller amount of the new diets with the low-dietary fibre diet that was being fed.

The pigs were fed three times daily at 08.00 hours (breakfast), 13.00 hours (lunch) and 18.00 (dinner). To mimic the diurnal variation of human cereal intake, the pigs were fed an amount of 40,40 and $20 \%$ of the daily supply at the respective feedings. On day 18 , the pigs were transferred to metabolic cages and urine samples were collected quantitatively throughout day 21 . To reduce dilution of urine samples caused by spillage of drinking water, water was only supplied ad libitum at meals during sample collection. Urine samples were stored at $-20^{\circ} \mathrm{C}$ immediately after sample collection and were permanently stored at $-80^{\circ} \mathrm{C}$.

\section{Analytical methods}

The diets were analysed in duplicate for DM, ash, protein (Kjeldahl method), fat (hydrochloric acid-fat), sugars, fructans, starch, total soluble and insoluble NSP and Klason lignin. Information on the chemical analysis procedures of the breads can be found in the studies by Le Gall et al. ${ }^{(25)}$ and Theil et al. ${ }^{(26)}$. Resistance starch was analysed enzymatically using the Megazyme assay (Megazyme International Limited). Analysis of the diets for plant lignans was completed using isotope dilution GC-MS in the selected ion monitoring mode (ID-GC-MS-SIM; Fisons Instrumentation, Inc.) as described by Peñalvo et al. ${ }^{(19)}$.
Analyses of plant lignans and enterolignans in urine and plasma samples were carried out on an UltiMate 3000 (Dionex; Thermo Scientific) HPLC system combined with a coulometric electrode array detection (CEAD) (Coularray; ESA). Urine samples were pre-treated according to Nurmi et al. ${ }^{(27)}$. In brief, ascorbic acid was added to the urine samples, and the samples were incubated with a hydrolysis reagent ( $\beta$-glucuronidase from Helix pomatia) for $16 \mathrm{~h}$. Lignans were extracted with diethyl ether and purified using QAE-Ac-ion-exchange chromatography (Amersham Pharmacia Biotech AB). Plasma samples were stored in Na-heparin tubes and pre-treated as described by Peñalvo et al. ${ }^{(28)}$. The procedures described by Peñalvo et $a l .{ }^{(28)}$ and Nurmi et $a l .{ }^{(27)}$ were modified with an additional purification step. Therefore, both urine and plasma samples were passed through a $0 \cdot 2-\mu \mathrm{m}$ Nylon filter (Costar Spin-x HPLC; Corning Inc.) before loading onto the HPLC-CEAD. The lower limit of detection in plasma and urine of enterolignans has been estimated to be 0.9-1.6 and $2 \cdot 0-2 \cdot 5 \mathrm{nmol} / 1$ and the limit of quantification to be $4 \cdot 3-7 \cdot 8$ and $10 \cdot 1-12 \cdot 4 \mathrm{nmol} / 1$, respectively ${ }^{(27,28)}$. The limit of detection of plant lignans in plasma and urine has been estimated to be $0.8-1.4$ and $1.9-3.9 \mathrm{nmol} / \mathrm{l}$ and the limit of quantification to be 3.6-6.9 and 9.4-19.7 nmol/1, respectively. The CV of detected plant lignans and enterolignans form the plasma samples for each batch was $24 \cdot 2$ and $10 \cdot 9 \%$, respectively.

All lignan analyses included hydrolysis of any conjugate (glucosides in diets, glucoronides and sulphates in the plasma and urine), and therefore the results are expressed as aglycones.

\section{Calculations and statistical analysis}

The quantitative absorption of lignans in study 1 was calculated from the porto-arterial differences and the portal flow measurements as described by Rérat et $a l .{ }^{(29)}$ :

$$
q=\left(C_{p}-C_{a}\right) F(d t), Q=\Sigma q,
$$

where $q$ is the amount of a given lignan net absorbed within the time period $d t, C_{p}$ the concentration of a given lignan in the portal vein, $C_{a}$ the concentration of a given lignan in the mesenteric artery, $F$ the blood flow in the portal vein and $Q$ the amount of a given lignan net absorbed from $t_{0}$ to $t_{1}$.

Urinary excretion of lignans in study 2 was calculated as the collected daily volume of urine ( $V_{\text {urine, }}, \mathrm{g}$ ) multiplied by the concentration of plant lignans and enterolignans, respectively, in urine samples expressed in $\mu \mathrm{mol} / \mathrm{g}$ :

$$
\text { Total plant lignan excretion }\left(\frac{\mu \mathrm{mol}}{\mathrm{d}}\right)=\frac{V_{\text {urine }}(g) \times T_{\text {plant lignan }}\left(\frac{\mathrm{nmol}}{\mathrm{l}}\right)}{1000 \times 1000}
$$

Total urinary excretion of enterolignans:

$$
\text { Total enterolignans }\left(\frac{\mu \mathrm{mol}}{\mathrm{d}}\right)=\frac{V_{\text {urine }}(g) \times T_{\text {enterolignan }}\left(\frac{\mathrm{nmol}}{\mathrm{l}}\right)}{1000 \times 1000} .
$$

Data were analysed using JMP ${ }^{\circledR}$; version 11.1.1 (SAS Institute Inc.), 1989-2007, selecting standard least squares as personality within the fit model platform. 
Fasting plasma variables after switching from diet WFL to the lignan-rich diets (days 4-7) in study 1 were analysed using the following mixed model:

$$
Y_{i j k l}=\mu+\alpha_{i}+\beta_{j}+\alpha \beta_{i j}+\delta_{k}+\rho_{l}+v_{k l}+e_{i j k l},
$$

where $Y_{i j k l}$ is the dependent variable, $\alpha_{i}$ the bread ( $i=\mathrm{WWG}$, WAF or RAF); $\beta_{j}$ the time after switching from diet WFL to one of the lignan-rich diets (i.e. WWG, WAF, RAF), $\alpha \beta_{i j}$ the two-factor interaction term, $\delta_{k}$ the week $(k=1,2$ or 3$), \rho_{1}$ the pig ( $l=$ pig $1, \ldots, 6)$ and $v_{k l}$ accounted for repeated measurements being performed on the same pig $\left(\rho_{l}\right)$ within period $\left(v_{k l}\right)$, whereas $e_{i j k l}$ describes the random error. The random effects were estimated using the restricted maximum likelihood method in $\mathrm{JMP}^{\circledR}$; version 11.1.1; insignificant interactions were removed from the analyses and the statistical model was re-analysed.

The plasma variables on the profile day (day 7) in study 1 were analysed using the following mixed model:

$$
\begin{gathered}
Y_{i j k l m n}=\mu+\alpha_{i}+\beta_{j}+\gamma_{k}+\alpha \beta_{i j}+\alpha \gamma_{i k}+\beta \gamma_{j k} \\
+\delta_{l}+\rho_{m},+v_{i l m}+\tau_{i l m n}+e_{i j k l m n},
\end{gathered}
$$

where $Y_{i j k l m n}$ is the dependent variable, $\alpha_{i}$ the bread ( $i=$ WWG, WAF or RAF), $\beta_{j}$ the effect of meal ( $j=1$ or 2$)$, which was also associated with length of time without feed before the meal ( 14 and $5 \mathrm{~h}$, respectively) (note that the third meal within a day was not studied), $\gamma_{k}$ the two phases after a meal $(k=1$ and 2 refer to $0-2.5$ and $2.5-5 \mathrm{~h}$ after feeding, respectively), $\alpha \beta_{i j}, \alpha \gamma_{i k}$ and $\beta \gamma_{j k}$ are two-factor interaction terms, $\delta_{l}$ is the week $(l=1$, 2 or 3$)$ and $\rho_{m}$ the pig $(m=$ pig $1, \ldots, 6) ; v_{i l m}$ and $\tau_{i l m n}$ ( $n$ meal 1,2 ) accounted for repeated measurements being performed on the same pig $\left(\rho_{m}\right)$, on the same pig within period $\left(v_{i l m}\right)$ and on the same pigs within period and meal $\left(\tau_{i l m n}\right)$, respectively, whereas $e_{i j k l m n}$ describes the random error. The random effects were estimated as described above. If $P>0.05$ was found for bread $\times$ meal, meal $\times$ phase or bread $\times$ phase, the non-significant interaction terms were removed from the analyses and the statistical model was re-analysed.

The fluctuations in plasma concentrations of plant lignans and enterolignans after switching to the lignan-rich diets (days 4-7) and on the profile day (day 7) were described by a mechanistic growth model:

$$
Y=\alpha\left(1-\beta e^{-c T}\right),
$$

where $y$ is the dependent variable (plant lignans or enterolignans, in the portal vein and the mesenteric artery, respectively), $\alpha$ is the asymptotic value, $c$ is the growth rate, $\beta$ is the scale and $T$ is time.

A one-way ANOVA model was used to analyse the urinary concentration and excretion data after log-transformation before the statistical analysis. The logarithmic transformations were applied to obtain normality and to stabilise the variances; both the normality and the variance homogeneity were informally verified by examining the residual plots. $F$ tests for nested models were applied to test equality of diets.

In all analyses, the level of significance was $P<0 \cdot 05$, whereas a tendency was reported when $P$ values were between 0.05 and $0 \cdot 1$.

\section{Results}

Diets

The diets were composed to provide equal amounts of starch, protein, fat, dietary fibre and energy, which was successfully accomplished (Table 2). Among the diets, there was a substantial variation in the concentration of plant lignans. The lowest concentration was found in the WFL diet $(1860 \mu \mathrm{g} / \mathrm{kg} \mathrm{DM})$, which had a 5-8-fold lower plant lignan concentration than the other diets. Among the lignan-rich diets, the concentration of plant lignans was the highest in the two diets based on aleurone-rich ingredients - WAF $(14970 \mu \mathrm{g} / \mathrm{kg} \mathrm{DM})$ and RAF $(13170 \mu \mathrm{g} / \mathrm{kg} \mathrm{DM})$ - compared with WWG $(9230 \mu \mathrm{g} / \mathrm{kg} \mathrm{DM})$.

\section{Effect of diet on arterial and portal vein enterolignan concentrations and absorption at fast}

The intake of plant lignans when consuming the WFL diet was $6.7 \mu \mathrm{mol} / \mathrm{d}$, whereas the intake when consuming the lignan-rich diets was 38.0-56.4 $\mu \mathrm{mol} / \mathrm{d}$. The animals were fed WFL diet on day 1-3, and fasting blood samples collected before breakfast on day 4 showed that enterolignan plasma concentrations were indeed reduced to a minimum when the WFL diet was provided $-18.1 \mathrm{nmol} / \mathrm{l}$ in the portal vein and $13.6 \mathrm{nmol} / 1$ in the mesenteric artery, respectively (Table 3). When the animals switched to the lignan-rich diets, the mean enterolignan concentration increased $(P<0.0001)$ to $90($ SEM 8.5$) \mathrm{nmol} / \mathrm{l}$ in the portal vein and to $64(\operatorname{sem} 5 \cdot 6) \mathrm{nmol} / \mathrm{l}$ in the mesenteric artery, respectively, on day 7 (Table 3 ). No difference was seen between the lignan-rich diets, but all three diets caused higher enterolignan concentrations in the mesenteric artery and portal vein. The change from day 4 to 7 in plasma concentrations of enterolignans after switching to the lignan-rich diets could be described by a mechanistic growth model with estimated growth rates of 0.0175 and $0.0124 \mathrm{nmol} / 1$ per $\mathrm{h}$ in the portal vein and mesenteric artery, respectively (Fig. 1).

In contrast, plant lignan concentration in the blood at fast was constant and in most cases below the limit of detection from day 4 to 7 , when measured in the portal vein and in the mesenteric artery.

The net absorption of enterolignans increased 6-fold (time effect $P<0.0017$ when switching from the WFL diet to the lignan-rich diets but with no difference between the lignan-rich diets. The response in plant lignans was more variable and with no specific trend compared with the WFL diet; furthermore, the measured plant lignan concentrations were below limit of detection.

\section{Net portal absorption of plant lignans and enterolignans during a 10-h postprandial profile}

The 10-h blood profile completed on day 7 presents the variation in plant lignan and enterolignan levels after breakfast and lunch. Breakfast was provided after a 14-h fast and lunch after a 5-h fast, and at both meals pigs received on average $57 \mu \mathrm{mol}$ of plant lignans. At fast, plant lignan levels were below the limit of detection in the mesenteric artery and the portal vein 
Table 2. Chemical composition of the diets (per kg DM)

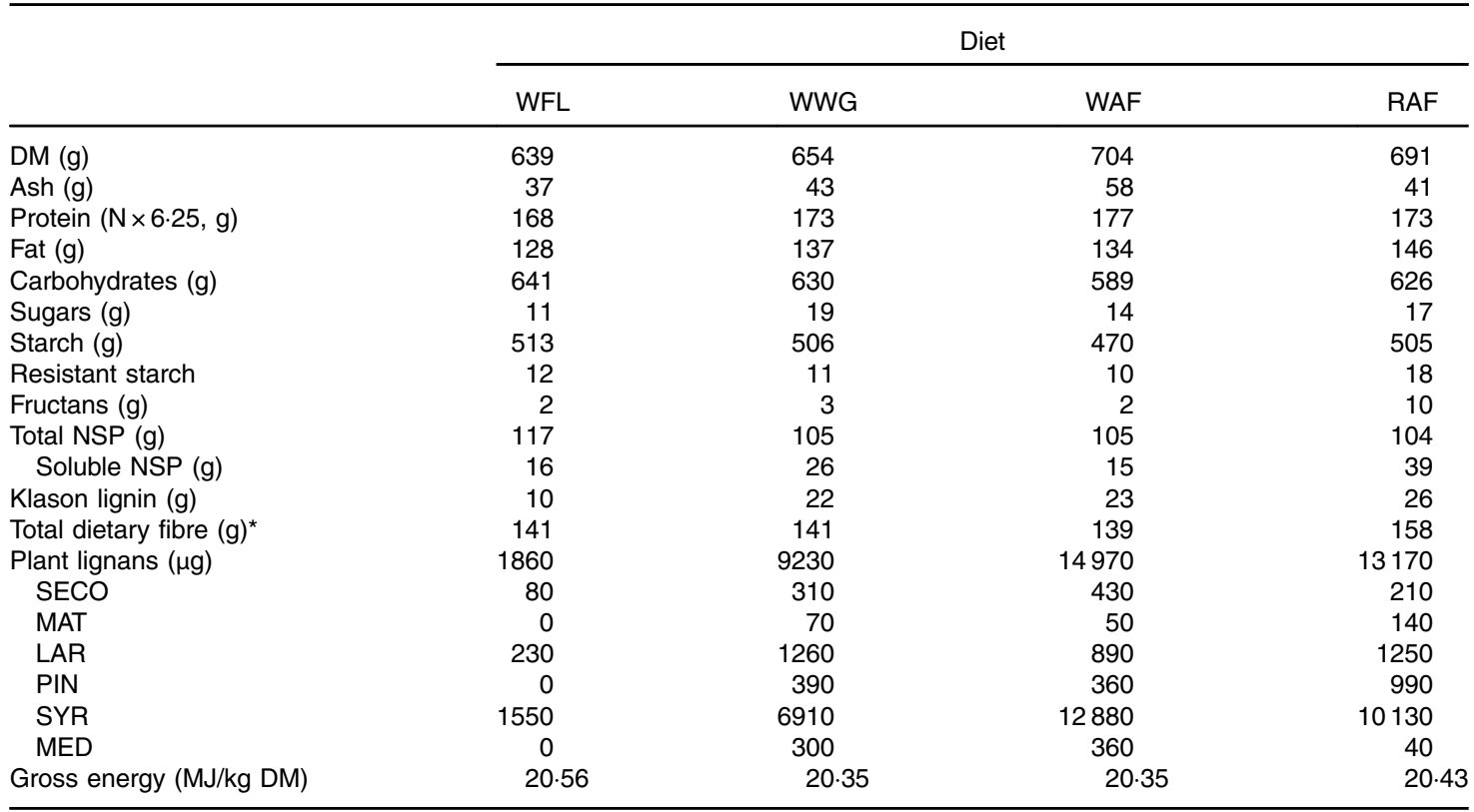

WFL, wheat flour; WWG, whole-wheat grain; WAF, wheat aleurone-rich flour; RAF, rye aleurone-rich flour; SECO, secoisolaricinol; MAT, matairesinol; LAR, lariciresinol; PIN, pinoresinol; SYR, syringaresinol; MED, medioresinol.

* Total dietary fibre: sum of total NSP, fructans, resistant starch, Klason lignin.

(approximately 1.5 and $2 \cdot 0 \mathrm{nmol} / 1$, respectively). In Phase I $(0-2.5 \mathrm{~h})$, a rapid increase in plant lignan concentration was observed at both sampling sites, and plant lignan concentrations continued to increase through Phase II $(2 \cdot 5-5 \mathrm{~h})$, reaching a level approximately seven times higher than fasting levels (Table 4). After lunch, the plant lignan concentration in the artery increased in Phase III $(5-7.5 \mathrm{~h})$, but the concentration did not increase further in Phase IV $(7 \cdot 5-10 \mathrm{~h})$. In the portal vein, plant lignan levels found in Phase II were maintained through Phase III and Phase IV. The statistic model showed interaction between phase and meal, resulting from uneven changes in plant lignan concentration following breakfast and lunch. The fluctuations in plant lignan concentrations both in the artery and in the vein in the time frame $0-10 \mathrm{~h}$ after the first meal (breakfast) could be described by a mechanistic growth model with growth rates of 0.404 and $0.260 \mathrm{nmol} / \mathrm{l}$ per $\mathrm{h}$ in the portal vein and the mesenteric artery, respectively (Fig. 2).

The increased concentration of plant lignans in the portal vein after breakfast and lunch was followed by a concomitant increase in the concentration of plant lignans in the peripheral circulation; the correlation between the concentrations at the two sampling sites was $0.933(P<0.0001)$.

Although the plant lignan concentrations increased rapidly after breakfast, a concomitant decrease in enterolignan plasma concentration was observed, resulting in the lowest level $2 \cdot 5-5 \cdot 0 \mathrm{~h}$ after breakfast at both sampling sites (Table 4). Although there was a rebound after lunch, the concentration neither in the mesenteric artery nor in the portal vein reached the concentrations at fast.

The blood-flow rate in the portal vein was not affected by the dietary treatment, but fluctuated with time after feeding.
The net absorption of enterolignans at fast was estimated to be 1478 (sEM 171) and 938-1306 (sEM 207) nmol/h during the 10-h sampling period. Owing to large inter-individual variation, no significant differences were observed in lignan absorption neither between diets nor among the different phases. However, the net absorption of plant lignans increased almost 10 -fold after breakfast relative to fasting with no further increase during the day. During the 10-h sampling period, the cumulated net absorption of plant lignans amounted to $4 \cdot 1 \mu \mathrm{mol}$, corresponding to $7-8 \%$ of ingested plant lignan. Analysis of the cumulative absorption of the individual plant lignans was performed; however, no specific trend was observed, most likely due to large inter-individual variation. At fast, plant lignans represented $2 \%$ of the total absorbed lignans compared with $21-32 \%$ of plant lignans after feeding.

\section{Dietary intake of nutrients and urinary excretion}

The intake of plant lignans is shown in Table 5 along with the urinary concentrations and excretion of plant lignans and enterolignans. Consumption of the WFL diet resulted in an intake of plant lignans of $8.1 \mu \mathrm{mol} / \mathrm{d}$, which increased to $40 \cdot 1-63.9 \mu \mathrm{mol} / \mathrm{d}$ when consuming lignan-rich diets. The higher intake of plant lignans via the lignan-rich diets compared with WFL gave rise to a significantly higher urinary concentration and excretion of plant lignans and enterolignans compared with the WFL diet, but with no difference between the lignan-rich diets, primarily due to high individual variation. Across diets, the urinary content of plant lignans was $3 \%$ of the ingested plant lignans, whereas concentrations of enterolignans were 8.5-22 times higher (Table 5). 


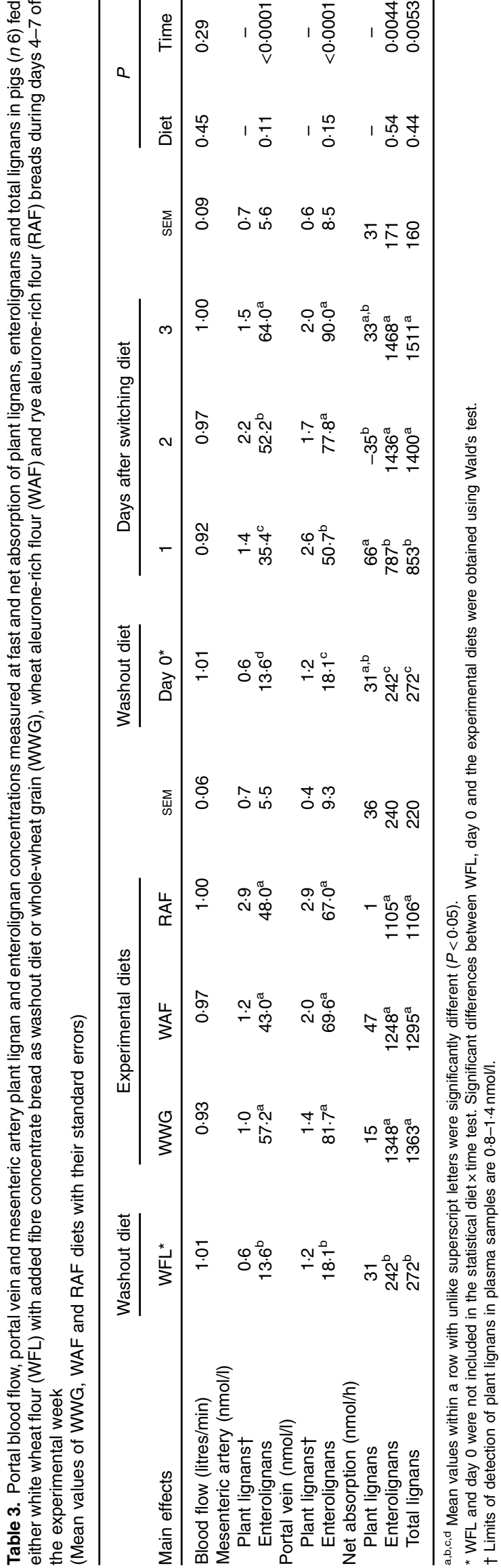

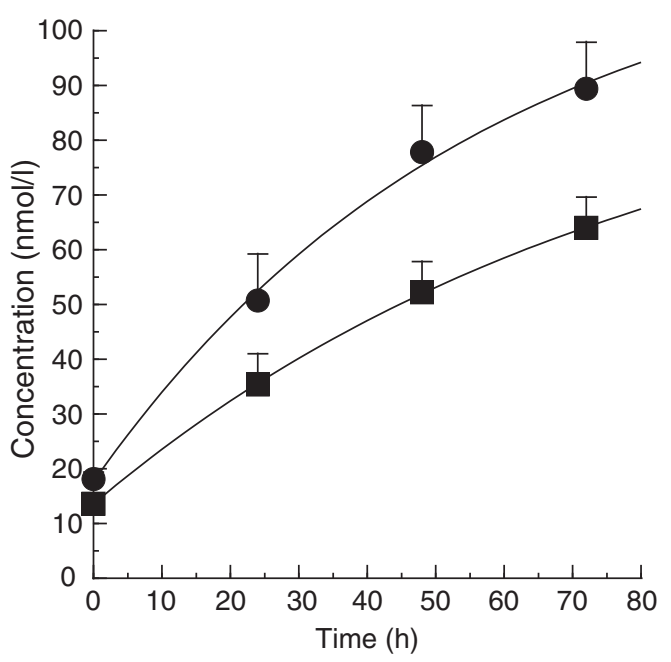

Fig. 1. Portal and arterial enterolignan plasma concentrations at fast during days 4-7 of the experimental week after switching from the low-lignan wheat flour (WFL) diet (time 0 ) to the lignan-rich diets (wheat-whole grain (WWG), wheat aleuronerich flour (WAF), rye aleurone-rich flour (RAF)). Plasma concentrations are presented as means resulting from intakes of WWG, WAF or RAF, with their standard errors represented by vertical bars. The variation in plasma concentration in the mesenteric artery (MA, could be described as follows: enterolignan $_{\mathrm{MA}}=99.0 \times(1-0.863 \times \operatorname{EXP}(-0.0124 \times T)), R^{2} 0.999$; and in the portal vein $(P V, O)$ by: enterolignan $P V=119.2 \times(1-0.852 \times \operatorname{EXP}(-0.0175 \times T)$ ), $R^{2}$ 0.997, where $T$ is the time after switching to the lignan-rich diets.

In spite of the high individual variation, a strong positive relationship between intake of plant lignans and urinary excretion of total lignans was found:

Total lignans $(\mu \mathrm{mol} / \mathrm{d})=0.85+0.40 \times$ plant lignan intake $(\mu \mathrm{mol} / \mathrm{d}), R^{2} 0.9822$.

\section{Discussion}

The most significant finding of the present investigation was the substantial and rapid uptake of plant lignans after breakfast and lunch measured in female pigs. A substantial rise was seen in the concentration of plant lignans as early as $0-2.5 \mathrm{~h}$ after breakfast and with a continuous build up in concentration over the following phases. This is also documented by the growth parameters in the mechanistic growth model that was twenty-one to twenty-three times higher for plant lignans than for enterolignans. Although we have limited knowledge on the mechanism by which plant lignans are taken up, a recent in vitro study reported that SECO exhibit low-to-moderate permeability at the intestinal barrier and are transported by passive diffusion $^{(30)}$. The conditions after a long fasting period favour a rapid absorption of plant lignans by passive diffusion to the blood circulation due to high plant lignan gradient between the intestine lumen and the blood circulation. This is consistent with the more rapid rise in mesenteric artery and portal vein concentrations that occurred after breakfast than after lunch. Although the net absorption of plant lignans at fast only represented $2 \%$ of total lignans, the proportion after breakfast and lunch represented $21-32 \%$ of absorbed lignans along with a 
Absorption of plant lignans

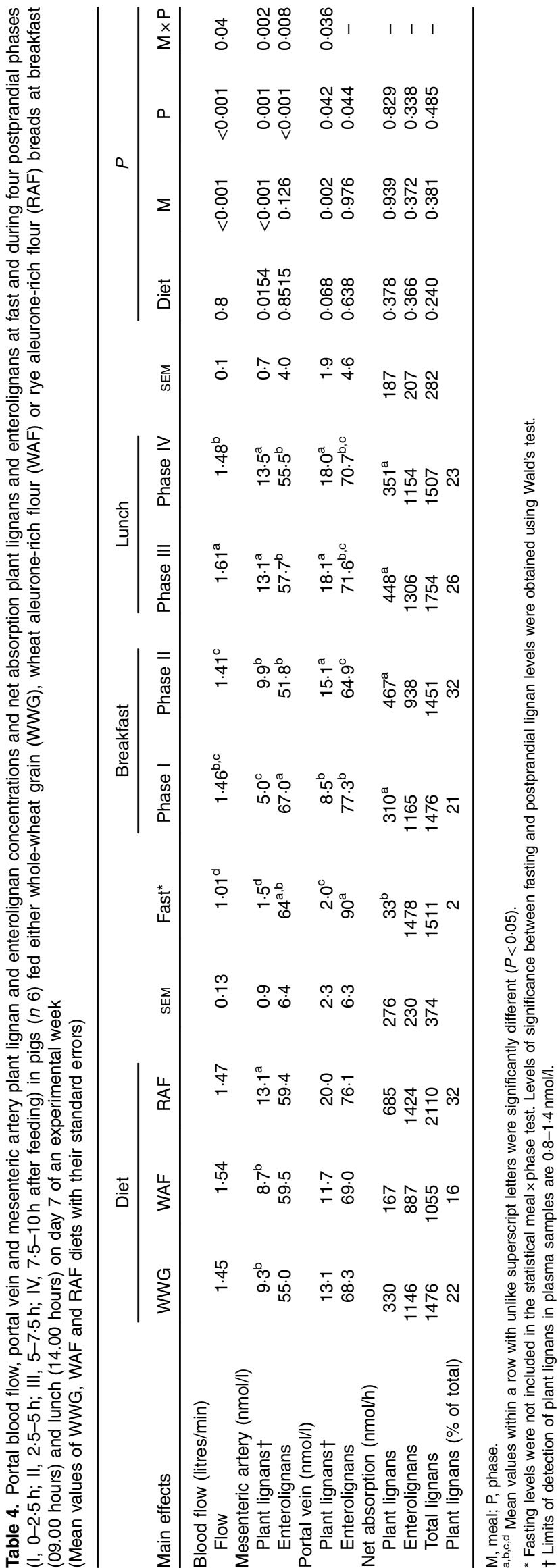

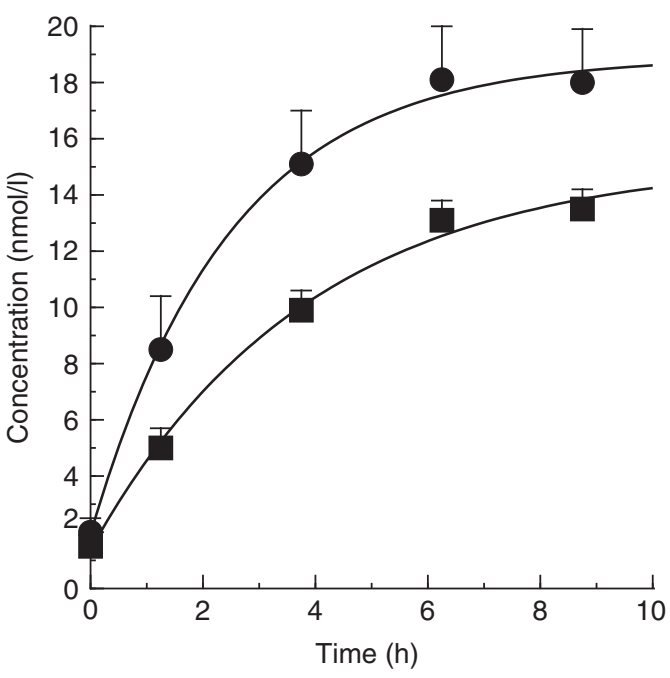

Fig. 2. Portal and arterial plant lignan plasma concentrations during day 7 of the experimental week after consuming the lignan-rich diets (wheat-whole grain (WWG), wheat aleurone-rich flour (WAF), rye aleurone-rich flour (RAF)) at breakfast and lunch. Plasma concentrations are presented as means resulting from intakes of WWG, WAF or RAF, with their standard errors represented by vertical bars. The variation in plasma concentration in the mesenteric artery (MA, $\square$ ) could be described as follows: plant $\operatorname{lignan}_{\mathrm{MA}}=15.3 \times(1-0.911 \times \mathrm{EXP}(-0.260 \times T))$, $R^{2}$ 0.99; and in the portal vein (PV, ) by: plant lignan ${ }_{\mathrm{PV}}=18.9 \times(1-0.898 \times \mathrm{EXP}$ $(-0.404 \times T)), R^{2} 0.997$, where $T$ is the time after breakfast.

concomitant decrease in the concentration of enterolignans. Over the 10-h sampling period, the cumulated net absorption of plant lignans corresponded to $7 \%$ of ingested plant lignans.

The rapid response in the uptake of plant lignans points to the small intestine as the site of absorption of plant lignans, as the concentration of plant lignans in both the portal vein and the mesenteric artery increased significantly in Phase I relative to the concentration at fast and gradually increased through the following sampling periods. In an earlier study, we found that it was not until $6 \mathrm{~h}$ after providing a pulse dose of lignan-rich bread that the concentration of enterolignans in the portal vein and mesenteric artery started to increase ${ }^{(10)}$.

The absorbed plant lignans are relatively rapidly cleared from the body; $14 \mathrm{~h}$ after dinner (20\% of the daily ration), the concentration of plant lignans in plasma returned to low levels, ranging between 0.6 and $2.6 \mathrm{nmol} / \mathrm{l}$. This is in contrast with enterolignans, where the net absorption at fast is high and is gradually increased with time after introduction of lignan-rich diets. The rapid clearance of plant lignans is in concert with the results of kinetic studies on humans fed a pulse dose of SDG concentrate $^{(31,32)}$ and sesamin ${ }^{(33)}$. In the study with SDG, the concentration of plant lignans increased rapidly after oral intake, peaked at 5-7 h and disappeared with half-lives of $4 \cdot 8 \mathrm{~h}$. For comparison, maximum serum concentrations of END and ENL were attained after $12-24$ and $24-36 \mathrm{~h}$, respectively, and with half-lives of 9.4 and $13 \cdot 2 \mathrm{~h}$. These relatively long residence times are suggested to be due to enterohepatic circulation of the enterolignans circulating the lignans from the intestine, excreting them through the bile ducts and back to the intestinal tract. In addition to increasing the residence time, the enterohepatic circulation is suggested to have a buffering effect, leveling out the diurnal fluctuation in microbial production in 
Table 5. Intake of plant lignans and urinary concentrations and excretions of plant and enterolignans in pigs $(n 5)$ fed either whole-wheat grain (WWG), wheat aleurone-rich flour (WAF) or rye aleurone-rich flour (RAF) breads (Mean values with their standard errors and $95 \%$ confidence intervals)

\begin{tabular}{|c|c|c|c|c|c|}
\hline & \multicolumn{4}{|c|}{ Diet } & \multirow[b]{2}{*}{$P$} \\
\hline & WFL & WWG & WAF & RAF & \\
\hline Intake plant lignans $(\mu \mathrm{mol} / \mathrm{d})$ & $8 \cdot 1$ & $40 \cdot 1$ & 63.9 & $57 \cdot 0$ & \\
\hline \multicolumn{6}{|l|}{ Urinary concentration $(\mu \mathrm{mol} / \mathrm{l})$} \\
\hline Plant lignans & & & & & $<0.001$ \\
\hline Mean & $0 \cdot 1^{\mathrm{a}}$ & $0.3^{b}$ & $0.2^{b}$ & $0.4^{\mathrm{b}}$ & \\
\hline $95 \% \mathrm{Cl}$ & $0.0,0.2$ & $0.2,0.7$ & $0.1,0.5$ & $0.2,0.9$ & \\
\hline Enterolignans & & & & & $<0.01$ \\
\hline Mean & $0.8^{a}$ & $4.9^{\mathrm{b}}$ & $4.9^{\mathrm{b}}$ & $5 \cdot 7^{\mathrm{b}}$ & \\
\hline $95 \% \mathrm{Cl}$ & $0.4,1.9$ & $1.9,12.5$ & $1 \cdot 9,12 \cdot 3$ & $2 \cdot 5,13 \cdot 1$ & \\
\hline \multicolumn{6}{|l|}{ Urinary excretion ( $\mu \mathrm{mol})$} \\
\hline Plant lignans & & & & & $<0.01$ \\
\hline Mean & $0.4^{\mathrm{a}}$ & $1 \cdot 1^{\mathrm{b}}$ & 1.1 & $1.8^{b}$ & \\
\hline $95 \% \mathrm{Cl}$ & $0.2,0.6$ & $0.6,1.9$ & $0.6,2 \cdot 0$ & $1 \cdot 1,3 \cdot 0$ & \\
\hline Enterolignans & & & & & $<0.001$ \\
\hline Mean & $3.4^{\mathrm{a}}$ & $16 \cdot 1^{\mathrm{b}}$ & $23 \cdot 7^{\mathrm{b}}$ & $23 \cdot 5^{\mathrm{b}}$ & \\
\hline $95 \% \mathrm{Cl}$ & $2 \cdot 3,5 \cdot 1$ & $10 \cdot 3,25 \cdot 3$ & $15 \cdot 1,37 \cdot 2$ & $15 \cdot 7,35 \cdot 1$ & \\
\hline \multicolumn{6}{|l|}{ Urinary excretion ( $\%$ of intake) } \\
\hline Plant lignans & 4.4 & $2 \cdot 7$ & 1.7 & 3.1 & \\
\hline Enterolignans & 41.9 & $40 \cdot 2$ & $37 \cdot 1$ & $41 \cdot 2$ & \\
\hline Total lignans & $46 \cdot 3$ & $42 \cdot 9$ & $38 \cdot 8$ & $44 \cdot 3$ & \\
\hline
\end{tabular}

a,b Mean values within a row with unlike superscript letters were significantly different.

the gut ${ }^{(10)}$. This study as well as previous studies ${ }^{(10,11)}$ show that it takes a relatively long time until the plateau levels of circulating enterolignans are reached.

In this study, we found that plant lignans are absorbed from the small intestine; however, only a minor fraction of the ingested plant lignans is excreted via urine (1-4\%), whereas approximately $40 \%$ of ingested plant lignans is excreted as enterolignans. This tempts us to believe that plant lignans absorbed from the small intestine enter the enterohepatic circulation, such as enterolignans, and make a second round (or multiple) through the small intestine, continuing into the colon where the plant lignans are metabolised to enterolignans by the microbiota. The study of Lærke et al. ${ }^{(11)}$ supports this hypothesis by presenting contrasting proportion of plant lignans:enterolignans in the portal vein (influx to the liver), the bile (excretion to the gut) and the hepatic vein (outflow to the

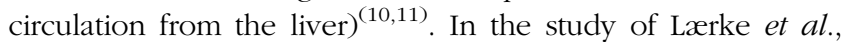
sampling $3 \mathrm{~h}$ after feeding showed that the proportion of plant lignans:enterolignans in the portal vein was $23: 77$, in the bile $77: 23$ and in the hepatic vein 17:83, suggesting that the liver, as a detoxification organ, strives to remove the absorbed plant lignans by excreting plant lignans to the intestine via the bile. Further support for the re-secretion of plant lignans from the body to the intestinal tract is the $2 \cdot 5$ to 10 times ileal recovery of ingested plant lignans ${ }^{(34)}$.

Although the biological activity of enterolignans, particularly in relation to cancer, has been discussed in several studies $^{(1,35,36)}$, much less is known about the biological function of plant lignans. In an in vitro study by $\mathrm{Hu}$ et $a{ }^{(37)}$, it was found that the antioxidant activities of flaxseed lignan SDG and its aglycone SECO were much higher than was the case with ENL or END. Although it can be difficult to extrapolate from an in vitro to an in vivo situation, the data of $\mathrm{Hu}$ et al. suggest that plant lignans could have health-promoting properties. In this context, it is interesting that the concentration of plant lignans is high not only in the portal vein but also in the mesenteric artery during the periods with high influx of nutrients to the body and high cellular activity ${ }^{(26)}$.

The data from the urinary excretion of plant lignans and enterolignans show that approximately $40 \%$ of ingested total lignans across diets was excreted via the urine; the remaining part will be excreted via faeces, or possibly accumulate in various tissues as demonstrated in rats ${ }^{(38)}$. In an earlier study where the full profile of plant lignans in diet and faeces was determined, total tract digestibilities of plant lignans of 80 and $83 \%$ were found for diets based on wheat and rye ${ }^{(11)}$. The study by Lærke et al. ${ }^{(11)}$, however, did not solubilise the faecal residue in an alkaline solution and plant lignan values in faeces were therefore most likely underestimated.

The urine excretion data for the three lignan-rich diets were similar, and we therefore expected similar effects on the arterial and the portal vein levels of enterolignans both in fasting blood samples and in blood samples obtained from the 10-h profile. This also applies for arterial and portal levels of plant lignans in fasting blood samples; however, during the 10-h profile, intake of RAF diet resulted in a significantly higher plant lignan concentration in the mesenteric artery compared with the intakes of WWG and WAF diets. Among the lignan-rich diets, RAF had an intermediate level of plant lignans, thus a higher plant lignan content in the diet cannot directly explain the elevated plasma level in the artery. RAF, on the other hand, had approximately three times higher level of PIN and two to three times higher MAT content compared with the other lignan-rich diets. Most likely, the absorption through the intestinal wall differs between the various types of plant lignans, resulting in a more or less rapid response of plant lignan concentrations in the blood 
according to the plant lignan composition of the diet fed. Mukker et $a l^{(30)}$ support this suggestion by concluding that SECO passively permeates across the intestinal epithelium in vitro, whereas SDG does not exhibit this ability ${ }^{(30)}$. This is possibly influenced by the chemical structure of the plant lignan and the hydrophobicity of the compound, where LAR and SECO differ from PIN and MAT in having at least one alcohol group present on the alkyl chain, whereas PIN and MAT have furan and lactone rings, respectively. Furthermore, Peñalvo et $a l .{ }^{(33)}$ reported a large variation in the time needed to reach maximum plasma concentrations for plant lignans, and thus PIN and MAT appearing approximately three and five times faster, respectively, in plasma compared with LAR and SECO. This could possibly explain the more rapid absorption of plant lignans in the RAF diet compared with WWG and WAF diets.

\section{Conclusion}

The major findings of the present study are the substantial and rapid absorption of plant lignans in the small intestine during the absorptive phases following intake of cereal-based diets. We demonstrated that plant lignan levels in plasma of female pigs increase rapidly after intake of lignan-rich cereal-based diets and, in contrast to enterolignans, are taken up from the small intestine.

\section{Acknowledgements}

The authors thank Helle Handll Christensen, Winnie Østergaard Thomsen, Kathrine Høirup Hansen and Lisbeth Märcher for technical assistance and Rodrigo Labouriau for excellent statistical advice.

The present research was financially supported by the European Commission in the Communities Sixth Framework Programme, Project HEALTHGRAIN (FOOD-CT-2005-514008). It reflects the authors' views, and the community is not liable for any use that may be made of the information contained in this publication.

K. E. B. K. was responsible for project development. P. K. T. and $\mathrm{H}$. J. conducted the nutritional experiments with pigs. A. K. B. was responsible for the analyses and for generating statistical analyses. A. K. B. and K. E. B. K. were responsible for drafting the manuscript.

There are no conflicts of interest.

\section{References}

1. Adlercreutz H (2007) Lignans and human health. Crit Rev Clin Lab Sci 44, 483-525.

2. Fardet A (2010) New hypotheses for the health-protective mechanisms of whole-grain cereals: what is beyond fibre? Nutr Res Rev 23, 65-134.

3. Bylund A, Lundin E, Zhang JX, et al. (2003) Randomised controlled short-term intervention pilot study on rye bran bread in prostate cancer. Eur J Cancer Prev 12, 407-415.

4. Jacobs DR Jr, Pereira MA, Stumpf K, et al. (2002) Whole grain food intake elevates serum enterolactone. Br J Nutr 88, 111-116.
5. Juntunen KS, Mazur WM, Liukkonen KH, et al. (2000) Consumption of wholemeal rye bread increases serum concentrations and urinary excretion of enterolactone compared with consumption of white wheat bread in healthy Finnish men and women. Br J Nutr 84, 839-846.

6. Vanharanta M, Voutilainen S, Nurmi T, et al. (2002) Association between low serum enterolactone and increased plasma F2-isoprostanes, a measure of lipid peroxidation. Atherosclerosis 160, 465-469.

7. Horner NK, Kristal AR, Prunty J, et al. (2002) Dietary determinants of plasma enterolactone. Cancer Epidemiol Biomarkers Preven 11, 121-126.

8. Johnsen NF, Hausner H, Olsen A, et al. (2004) Intake of whole grains and vegetables determines the plasma enterolactone concentration of Danish women. J Nutr 134, 2691-2697.

9. Kilkkinen A, Stumpf K, Pietinen P, et al. (2001) Determinants of serum enterolactone concentration. Am J Clin Nutr $\mathbf{7 3}$, 1094-1100.

10. Bach Knudsen KE, Serena A, Kjaer AK, et al. (2003) Rye bread in the diet of pigs enhances the formation of enterolactone and increases its levels in plasma, urine and feces. J Nutr $\mathbf{1 3 3}$, 1368-1375.

11. Laerke HN, Mortensen MA, Hedemann MS, et al. (2009) Quantitative aspects of the metabolism of lignans in pigs fed fibre-enriched rye and wheat bread. Br J Nutr 102, 985-994.

12. Björck I, Östman E, Kristensen M, et al. (2012) Cereal grains for nutrition and health benefits: overview of results from in vitro, animal and human studies in the HEALTHGRAIN project. Trends Food Sci Technol 25, 87-100.

13. Penttinen P, Jaehrling J, Damdimopoulos AE, et al. (2007) Diet-derived polyphenol metabolite enterolactone is a tissuespecific estrogen receptor activator. Endocrinology 148, 4875-4886.

14. Schöttner M, Ganßer D \& Spitelle G (1997) Interaction of lignans with human sex hormone binding globulin (SHBG). $Z$ Naturforsch C 52, 834-843.

15. Adlercreutz H, Höckerstedt K, Bannwart C, et al. (1987) Effect of dietary components, including lignans and phytoestrogens, on enterohepatic circulation and liver metabolism of estrogens and on sex hormone binding globulin (SHBG). I Steroid Biochem 27, 1135-1144.

16. Evans BAJ, Griffiths K \& Morton M (1995) Inhibition of $5 \alpha$-reductase in genital skin fibroblasts and prostate tissue by dietary lignans and isoflavonoids. J Endocrinol 147, 295-302.

17. Rickard SE, Yuan YV \& Thompson LU (2000) Plasma insulinlike growth factor I levels in rats are reduced by dietary supplementation of flaxseed or its lignan secoisolariciresinol diglycoside. Cancer Lett 161, 47-55.

18. Menendez JA, Vazquez-Martin A, Oliveras-Ferraros C, et al. (2008) Analyzing effects of extra-virgin olive oil polyphenols on breast cancer-associated fatty acid synthase protein expression using reverse-phase protein microarrays. Int J Mol Med 22, 433-439.

19. Peñalvo JL, Haajanen KM, Botting N, et al. (2005) Quantification of lignans in food using isotope dilution gas chromatography/mass spectrometry. J Agri Food Chem $\mathbf{5 3}$, 9342-9347.

20. Smeds AI, Eklund PC, Sjoholm RE, et al. (2007) Quantification of a broad spectrum of lignans in cereals, oilseeds, and nuts. J Agri Food Chem 55, 1337-1346.

21. Clavel T, Dore J \& Blaut M (2006) Bioavailability of lignans in human subjects. Nutr Res Rev 19, 187-196.

22. Clavel T, Lippman R, Gavini F, et al. (2007) Clostridium saccharogumia sp. nov. and Lactonifactor longoviformis gen. nov., sp. nov., two novel human faecal bacteria involved in 
the conversion of the dietary phytoestrogen secoisolariciresinol diglucoside. Syst Appl Microbiol 30, 16-26.

23. Kuijsten A, Arts IC, van't Veer P, et al. (2005) The relative bioavailability of enterolignans in humans is enhanced by milling and crushing of flaxseed. J Nutr $\mathbf{1 3 5}$, 2812-2816

24. Jørgensen H, Serena A, Theil PK, et al. (2010) Surgical techniques for quantitative nutrient digestion and absorption studies in the pig. Livest Sci 133, 57-60.

25. Le Gall M, Serena A, Jorgensen H, et al. (2009) The role of whole-wheat grain and wheat and rye ingredients on the digestion and fermentation processes in the gut - a model experiment with pigs. Br J Nutr 102, 1590-1600.

26. Theil PK, Jorgensen H, Serena A, et al. (2011) Products deriving from microbial fermentation are linked to insulinaemic response in pigs fed breads prepared from whole-wheat grain and wheat and rye ingredients. Br J Nutr 105, 373-383.

27. Nurmi T, Voutilainen S, Nyyssonen K, et al. (2003) Liquid chromatography method for plant and mammalian lignans in human urine. J Chromatogr B Analyt Technolo Biomed Life Sci 798, 101-110.

28. Peñalvo JL, Nurmi T, Haajanen K, et al. (2004) Determination of lignans in human plasma by liquid chromatography with coulometric electrode array detection. Analyt Biochem 332 384-393.

29. Rérat AA, Vaissade P \& Vaugelade P (1984) Absorption kinetics of some carbohydrates in conscious pigs. 2. Quantitative aspects. Br J Nutr 51, 517-529.
30. Mukker JK, Michel D, Muir AD, et al. (2014) Permeability and conjugative metabolism of flaxseed lignans by Caco- 2 human intestinal cells. J Nat Prod 77, 29-34.

31. Kuijsten A, Arts IC, Hollman PC, et al. (2006) Plasma enterolignans are associated with lower colorectal adenoma risk. Cancer Epidemiol Biomarkers Prev 15, 1132-1136.

32. Setchell KD, Brown NM, Zimmer-Nechemias L, et al. (2014) Metabolism of secoisolariciresinol-diglycoside the dietary precursor to the intestinally derived lignan enterolactone in humans. Food Funct 5, 491-501.

33. Peñalvo JL, Heinonen SM, Aura AM, et al. (2005) Dietary sesamin is converted to enterolactone in humans. J Nutr $\mathbf{1 3 5}$, 1056-1062.

34. Glitso LV, Mazur WM, Adlercreutz H, et al. (2000) Intestinal metabolism of rye lignans in pigs. Br J Nutr 84, 429-437.

35. Adlercreutz H (2002) Phyto-oestrogens and cancer. Lancet Oncol 3, 364-373.

36. Landete J (2012) Plant and mammalian lignans: a review of source, intake, metabolism, intestinal bacteria and health. Food Res Int 46, 410-424.

37. Hu C, Yuan YV \& Kitts DD (2007) Antioxidant activities of the flaxseed lignan secoisolariciresinol diglucoside, its aglycone secoisolariciresinol and the mammalian lignans enterodiol and enterolactone in vitro. Food Chem Toxicol 45 , 2219-2227.

38. Saarinen NM \& Thompson LU (2010) Prolonged administration of secoisolariciresinol diglycoside increases lignan excretion and alters lignan tissue distribution in adult male and female rats. BrJ Nutr 104, 833-841. 